\title{
A Study for Parametric Morphogeometric Operators to Assist the Detection of Keratoconus
}

\author{
Laurent Bataille 1,2,3,4 (iD), Francisco Cavas-Martínez ${ }^{1, *}$ (D), Daniel G. Fernández-Pacheco ${ }^{1}$ (iD), \\ Francisco J. F. Cañavate ${ }^{1}$ (D) and Jorge L. Alio ${ }^{3,4,5}$ \\ 1 Department of Graphical Expression, Technical University of Cartagena, 30202 Cartagena, Spain; \\ lbataille@vissum.com (L.B.); daniel.garcia@upct.es (D.G.F-P.); francisco.canavate@upct.es (F.J.F.C.) \\ 2 Department of Research and Development, Vissum Corporation Alicante, 03016 Alicante, Spain \\ 3 Keratoconus Unit of Vissum Corporation Alicante, 03016 Alicante, Spain; jlalio@vissum.com \\ 4 Department of Refractive Surgery, Vissum Corporation Alicante, 03016 Alicante, Spain \\ 5 Division of Ophthalmology, Miguel Hernández University of Elche, 03202 Alicante, Spain \\ * Correspondence: francisco.cavas@upct.es; Tel.: +34-968-338-856
}

Received: 14 November 2017; Accepted: 3 December 2017; Published: 5 December 2017

\begin{abstract}
The aim of this study is to describe a new keratoconus detection method based on the analysis of certain parametric morphogeometric operators extracted from a custom patient-specific three-dimensional (3D) model of the human cornea. A corneal geometric reconstruction is firstly performed using zonal functions and retrospective Scheimpflug tomography data from 107 eyes of 107 patients. The posterior corneal surface is later analysed using an optimised computational geometry technique and the morphology of healthy and keratoconic corneas is characterized by means of geometric variables. The performance of these variables as predictors of a new geometric marker is assessed through a receiver operating characteristic (ROC) curve analysis and their correlations are analysed through Pearson or Spearman coefficients. The posterior apex deviation variable shows the best keratoconus diagnosis capability. However, the strongest correlations in both healthy and pathological corneas are provided by the metrics directly related to the thickness as the sagittal plane area at the apex and the sagittal plane area at the minimum thickness point. A comparison of the screening of keratoconus provided by the Sirius topographer and the detection of corneal ectasia using the posterior apex deviation parameter is also performed, demonstrating the accuracy of this characterization as an effective marker of the diagnosis and ectatic disease progression.
\end{abstract}

Keywords: geometric modelling; cornea reconstruction; diagnosis; Scheimpflug; computer-aided design

\section{Introduction}

Nature confers a unique style and metric to the corneal structure, providing to its architecture several properties adapted to its functional requirements [1]. The cornea is the main refracting element and contributes to the aberrations on the human eye. A completely symmetrical structure would be free of coma, distortion, and chromatic aberration. However, three opposing characteristics can be seen due to the lack of symmetry: surfaces do not present symmetry of revolution, so they have deformations, misalignments, and decentralizations. For this reason, the analysis of the corneal geometry by studying the changes in its morphogeometric properties is fundamental to the assessment of vision quality and to improve the diagnosis of corneal pathologies [2].

One of this corneal disease is keratoconus, which is a corneal disorder where the paracentral or central area of the cornea undergoes progressive thinning and steepening, generating corneal scarring, corneal protrusion, and irregular astigmatism, which leads to a severe vision deterioration $[3,4]$. Thus, a significant corneal steepening is noticed in the anterior and posterior corneal surfaces and both curvatures are affected in keratoconic eyes [3,4]. Its incidence is estimated in 1:2000 of the general 
population, with a prevalence of 229:100,000 in Asia and 54:100,000 in the USA [4]. Prevalence of keratoconus is higher in areas with important exposure to ultraviolet light or by a combination of environmental and genetic factors [4].

In this aspect, several systems for diagnosing and classifying keratoconus severity are described in the literature. The majority of these grading systems have been elaborated by considering the patient's visual performance, corneal scarring, biomicroscopic signs, central corneal thickness, corneal keratometry readings, topographic morphology of the disease, and corneal aberrometry [5].

However, identifying and distinguishing the pathologic cases at its earliest grade have been the main goal for refractive surgeons because this is the main risk factor for ectasia after laser-assisted in situ keratomileusis (LASIK) [6]. The difficulty increases as there is not a clear limit defined between the earliest grades of keratoconus and a normal cornea. Despite this, it seems evident from recent investigations that changes in different corneal geometric parameters are detected when keratoconus begins to develop.

Another advance in this field is the introduction of Scheimpflug photography for topographic characterization of the cornea, which helps in the study and characterization of both anterior and posterior corneal surfaces [7]. Numerous quantitative geometric descriptors of the corneal surfaces for the diagnosis of ectasia and provided by corneal tomographers can be found in the scientific literature [5]. However, the values obtained for these descriptors vary depending on the tomographer used [8], preventing their widespread acceptance and clinical utility.

Recently, a group of world keratoconus experts gathered for the project "Global Consensus on Keratoconus and Ectatic Diseases" [9]. They defined a new concept called "ectasia progression", which describes progression of keratoconus in its different phases depending on the changes of magnitude of some singular points located at the posterior surface of the cornea, specifically the point of minimum thickness and the highest point (apex or point of maximum curvature) were both defined as the most significant. Nevertheless, none of the quantitative descriptors provided by the current corneal tomographers for the diagnosis of ectasias consider these singular points of the posterior corneal surface, so its quantitative description remains a challenge.

Geometric modelling of biological structures by using tools based on computer-aided design (CAD) is currently applied to disease diagnosis [10,11], design of medical devices [12,13], and geometric reconstruction for different purposes [14,15]. More specifically, in the field of ophthalmology, there are several studies that use CAD methods to develop the concept of a patient-specific model [16-20]. These patient-specific models are obtained from the so called "raw data" [21], which are generated by systems based on the projection of a slit of light onto the cornea and on the principle of the Scheimpflug photography [5]. These raw data are not interpolated and are used to generate a geometric model with the modal methods called Zernike polynomials. These Zernike polynomials are defined for all discrete points of the anterior and posterior corneal surfaces for their reconstruction. However, in cases of pathological corneas as, for instance, very aberrated corneas, it has been demonstrated that it is very difficult to define the Zernike polynomial order required to get the most relevant information about the corneal surfaces [21,22]. An alternative to these geometric models are the zonal methods called B-Spline functions [23]. However, this technology is not extended to the ophthalmology field, and it has only been used to generate customised models of the corneal biomechanics $[17,18,20]$. In these cases, the raw data obtained were incomplete in the periphery and the limbus areas due to extrinsic errors during the data acquisition process, so the authors had to interpolate data in order to obtain a complete geometric model. In the case of significant imperfections of the cornea, the data acquisition is difficult and a significant level of missing data could require the interpolation of important areas of the cornea resulting in a very ambiguous model. The development of methods to define in vivo customised geometric properties of the cornea based only on real raw data and without any kind of interpolation is extremely useful in clinical practice for the modelization of pathological corneas [21]. Therefore, a rational and objective keratoconus detection technique could be based on analysing the 
geometric morphometry of discrete landmarks in the region where the focused curvature was initially manifested on the posterior corneal surface.

This work presents a new concept of diagnosis based on geometric modelization of the cornea to improve the prognostic of corneal ectasia. The technique used in this study will create a 3D patient-specific customised model of the cornea and analyse different geometric parameters extracted from the posterior surface of the generated model to determine which of them could be defined as indicators of susceptibility to develop keratoconus (Figure 1). A validation study was conducted with healthy corneas and corneas diagnosed with keratoconus according to the Amsler-Krumeich (AK) grading system [4].

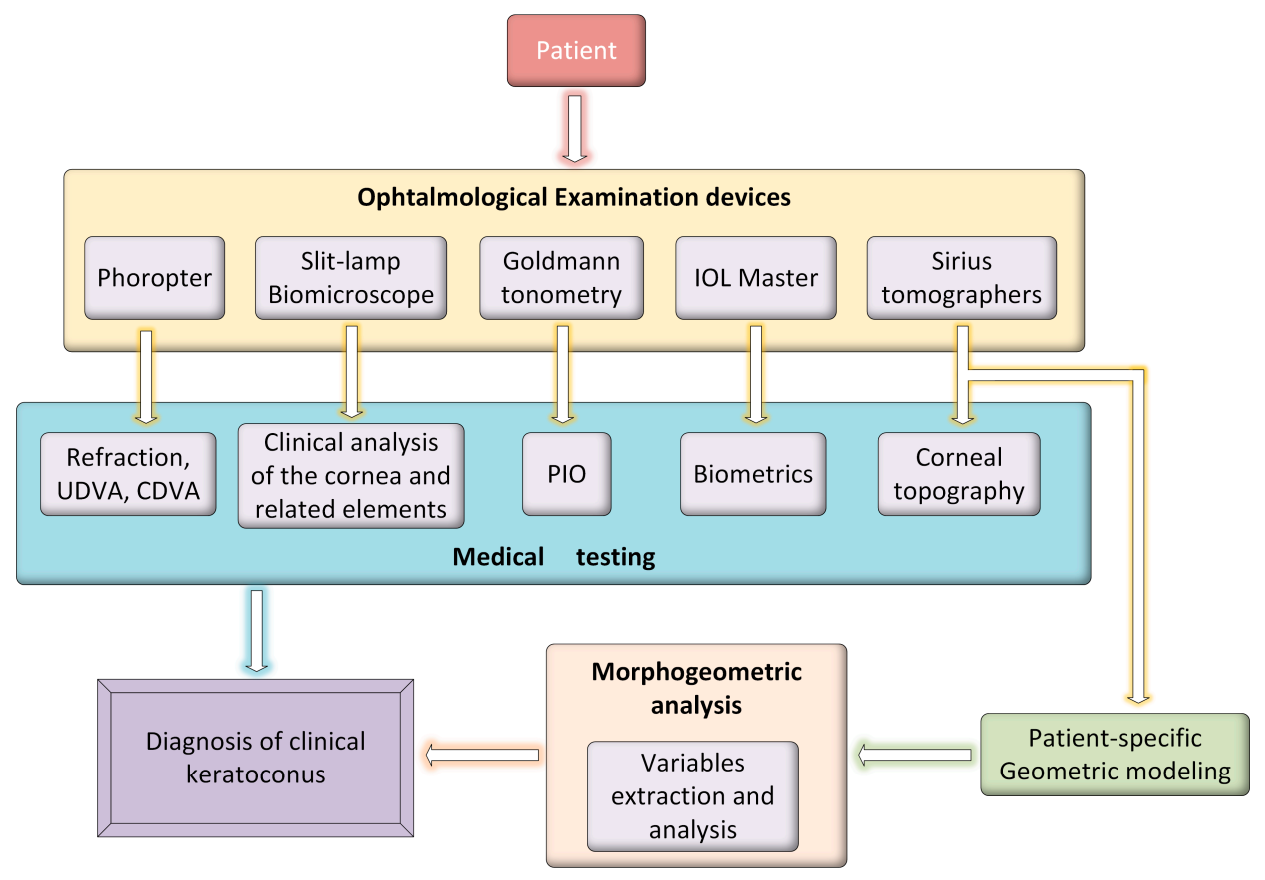

Figure 1. Use of patient-specific 3D modelling for the diagnosis of keratoconus.

\section{Materials and Methods}

\subsection{Patients}

The study comprised a total of 107 subjects admitted at Vissum Corporation in Alicante, Spain. All the procedures adhered to the Declaration of Helsinki and the approval of the Vissum Clinical Research Ethics Committee was obtained for this study (seventh revision, October 2013, Fortaleza, Brazil). Informed written consent for research and publication of the data was obtained from all patients before enrolment.

Patients were classified into two groups: a first group composed of 52 patients with keratoconic corneas and aged $17-73$ years $(39.11 \pm 16.70)$ and a second group of 55 patients with healthy corneas, aged 12-66 years (37.01 \pm 15.11$)$. Only one eye from each patient was randomly selected for the study according to a random number sequence (dichotomic sequence, 0 and 1 ). Specific software was developed for this process to avoid interference in the analysis of the correlation that often exists between the two eyes of the same person. The inclusion criteria for the keratoconus group was diagnosis of keratoconus based on standard guidelines $[4,24]$. Exclusion criteria in both groups were previous ocular surgery or any other active ocular disease. In order to obtain an accurate methodology only initial stages of the disease (stage I), according to Amsler-Krumeich grading, were used during this study. 


\subsection{Ophthalmological Examination}

A detailed and uniform ophthalmologic examination was performed in all cases. The examination included manifest refraction (sphere and cylinder), uncorrected distance visual acuity (UDVA), corrected distance visual acuity (CDVA), slit lamp biomicroscopy, Goldmann tonometry, fundus evaluation, tomographic analysis of anterior and posterior corneal surfaces by the Sirius system (CSO, Florence, Italy), and optical biometry by IOLMaster (Carl Zeiss Meditec AG, Jena, Germany). All measurements were performed by the same experienced technician. Regarding the corneal topographic assessment, three consecutive measurements were obtained to calculate the average values for posterior statistical analysis.

Data registration for the current study was performed with software suite Phoenix (CSO, v2.1, Florence, Italy).

\subsection{Morphogeometric Reconstruction of the Cornea}

The methodology proposed in this work starts with a first stage where a virtual 3D patient-specific model of the cornea is reconstructed from the raw data provided by a corneal tomographer (Figure 2). For this study, the Sirius system (CSO, Florence, Italy) was used jointly with the aid of the Phoenix v2.1 software from CSO, which permits to export the following data from the tomographer to a CSV file: raw non-interpolated altimetric data of the anterior/posterior elevations (in $\mu \mathrm{m}$ ) and radii of Placido's disc rings (in $\mathrm{mm}$ ) used during the capture process. Due to elevation data indicating the coordinates of each scanned point, two discrete and finite sets of spatial points representative of both anterior and posterior corneal surfaces, respectively, were then obtained.

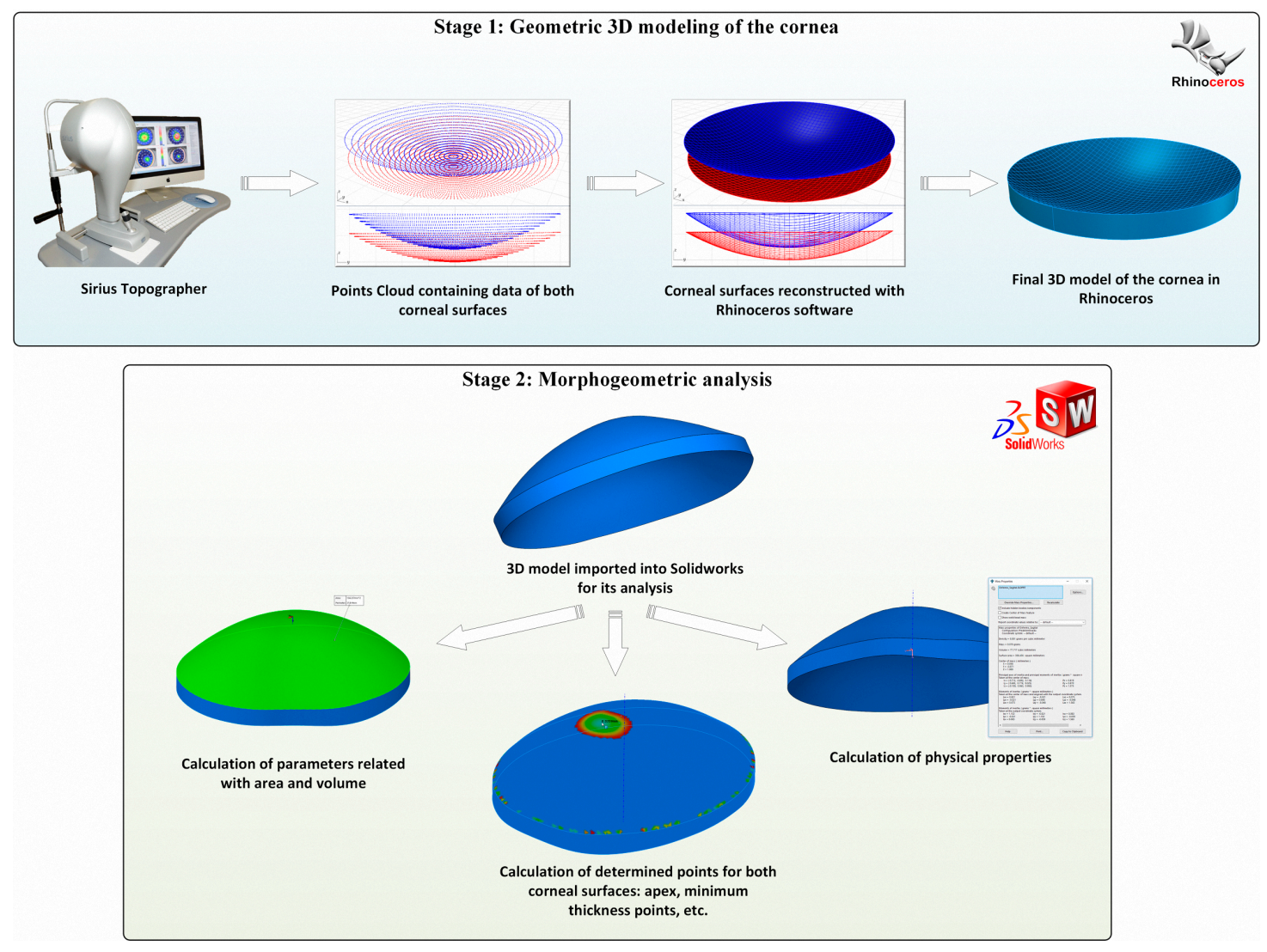

Figure 2. Geometric modelling process by using CAD tools.

In a second step (Figure 2), the point clouds representative of both corneal surfaces were imported into the surface reconstruction CAD software Rhinoceros ${ }^{\circledR}$ V 5.0 (McNeel and Associates, 
Seattle, WA, USA) [25]. This software enables to use non-uniform rational B-spline (NURBS) functions to generate reconstruction surfaces [26,27]. In this study, the Rhinoceros' surface from the point grid function was applied to the imported point cloud, creating a rectangular grid of 21 rows and 256 columns that was deformed to minimise the nominal distance between the spatial points and the grid surface. This deviation can be later calculated by the software, providing a mean value of the distance error for the solution surface. This can be seen in Figure 3, where the top view of the point cloud for the posterior surface of a cornea with keratoconus (stage I-AK) is represented and a mean distance error of $5.587 \times 10^{-16} \pm 5.76 \times 10^{-16} \mathrm{~mm}$ (mean \pm standard deviation) is obtained. In the figure, the following good/bad threshold values are configured: $5 \times 10^{-15} \mathrm{~mm}$ for bad points (in red) and $5.58 \times 10^{-16} \mathrm{~mm}$ for good points (in blue). The figure demonstrates that no point provides a distance greater than $5 \times 10^{-15}$ with respect to the solution surface, ensuring the accuracy of the surface.

By using this procedure, the anterior and posterior corneal surfaces were generated and connected by their geometrical centre in relation to the Z-axis (axis normal to the corneal vertex). Both surfaces and the peripheral surface (the bonding surface between both sides in the Z-axis direction) were then joined to obtain the final 3D solid model of the studied cornea.

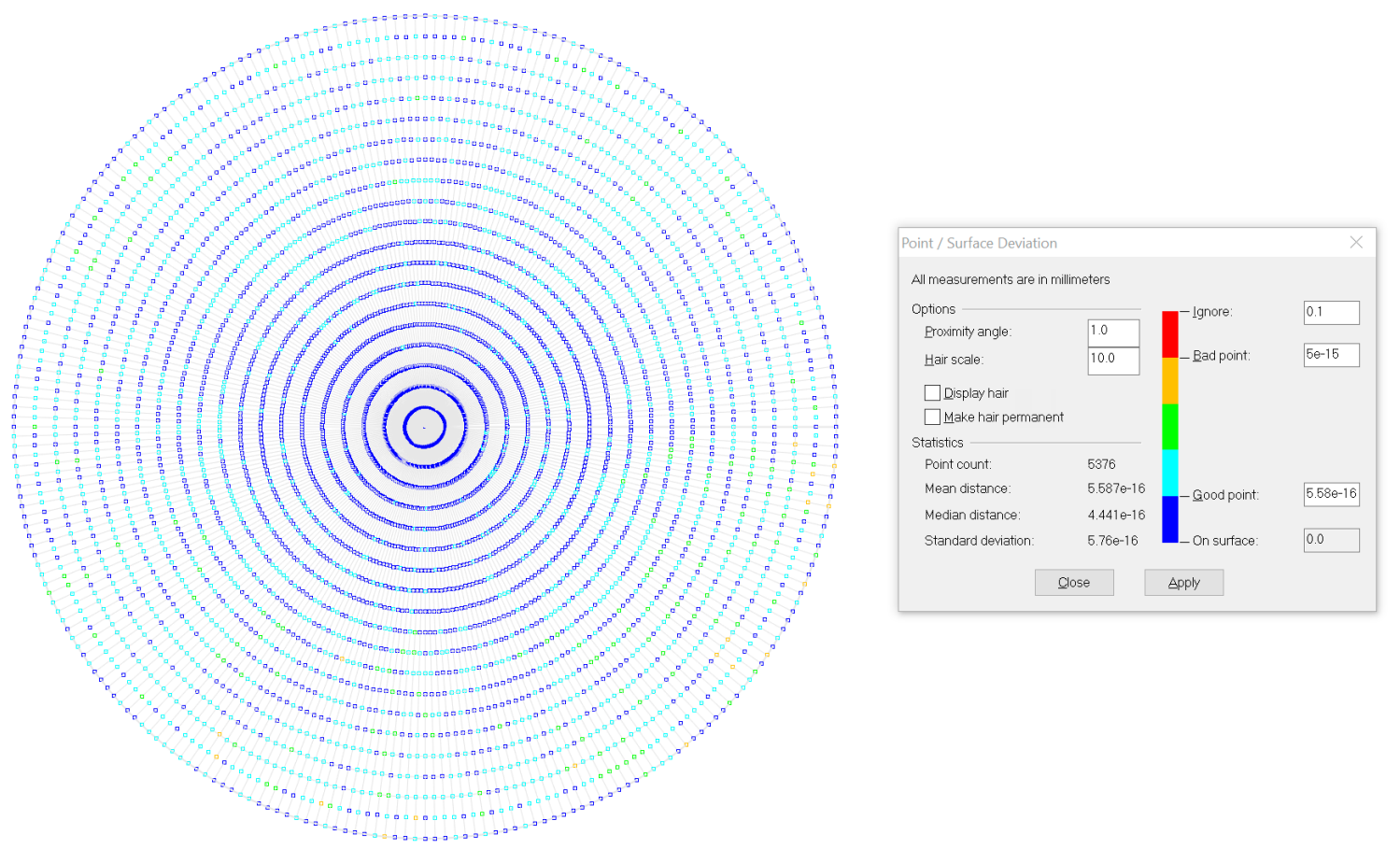

Figure 3. Analysis of the point-surface deviation for the posterior surface reconstruction of a cornea with keratoconus (stage I-AK).

\subsection{Morphogeometric Analysis}

The custom 3D model of the cornea generated in Rhinoceros is then exported to the solid modelling software SolidWorks (Dassault Systèmes, v2016, Vélizy-Villacoublay, France) [28,29], which permits the performance of a detailed analysis of the virtual model and the characterization of its morphology (Figure 2).

During this stage, singular points of the posterior surface were identified on each solid corneal model (healthy or keratoconic), and a morphogeometric analysis was performed in the local region [30,31] where curving (caused by progression of keratoconus) manifests gradually.

For this study, the following variables from the posterior surface of the cornea were analysed [32,33] (Figure 4): (i) the sagittal plane apex area, defined as the area of the cornea within the sagittal plane that passes through the highest point (apex, maximum curvature) of the posterior corneal surface and 
the Z-axis; (ii) the posterior apex deviation, defined as the average distance from the highest point (apex, maximum curvature) of the posterior corneal surface to the Z-axis; (iii) the sagittal plane area at the minimum thickness point, defined as the area of the cornea within the sagittal plane that passes through the minimum thickness point of the posterior corneal surface and the Z-axis; and (iv) the posterior minimum thickness point deviation, described as the average distance in the $X Y$ plane from the minimum thickness point of the posterior corneal surface to the Z-axis.
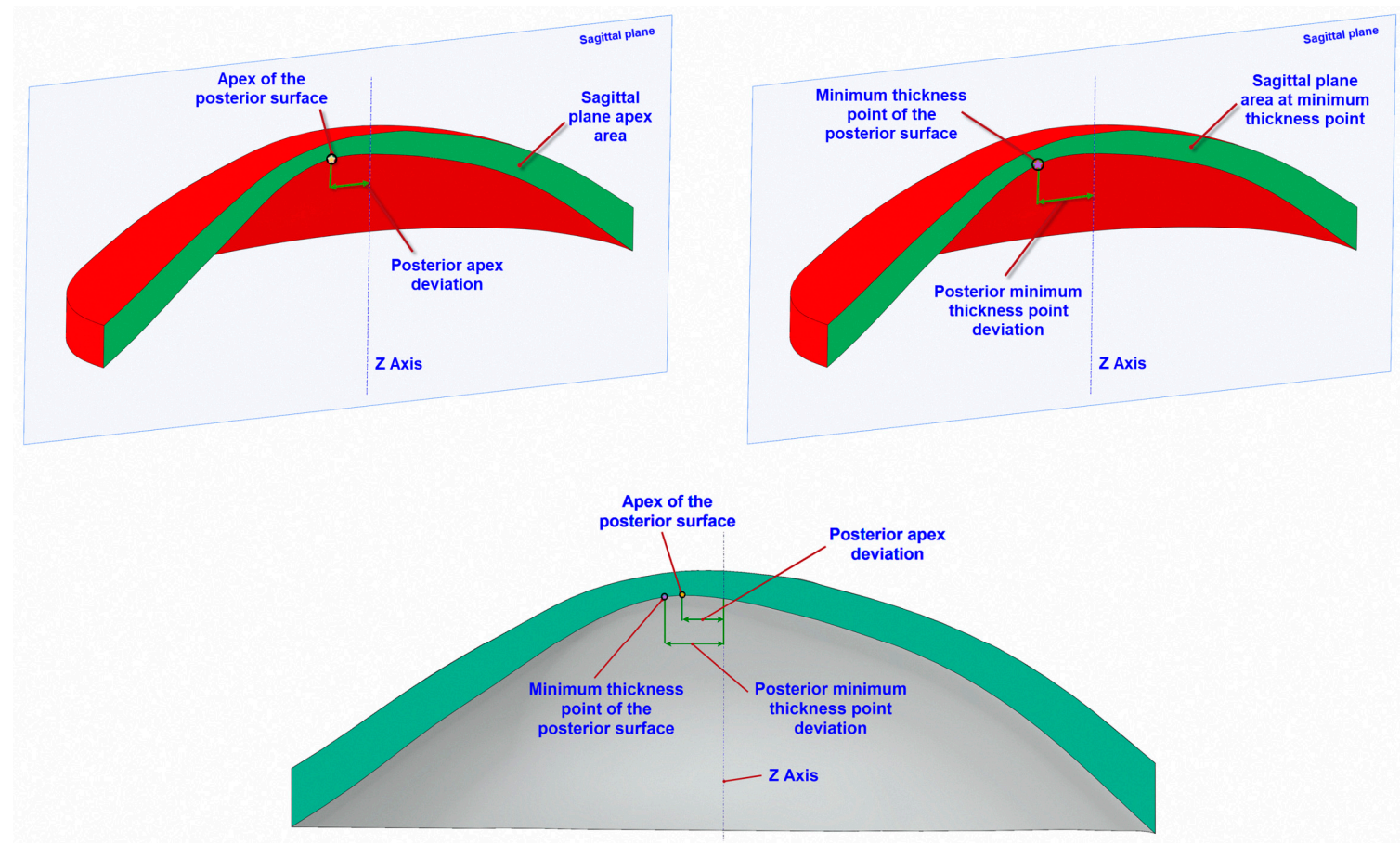

Figure 4. Geometric variables analysed during the study.

For each cornea, all these variables were measured by the same and unique observer using the SolidWorks caliper function.

\subsection{Statistical Analysis}

Data distribution was confirmed by means of the Kolmogorov-Smirnov test. According to this analysis, Student's $t$ and Mann-Whitney U tests were performed (depending on normality) in order to test the hypothesis according to the aim of the study. Additionally, effect size (ES) tests were used to compare differences and to quantify the degree of change between the healthy group and the stage I-KC group (according to Amsler-Krumeich Grading System). For all statistical tests, the same level of significance was used $(p<0.05)$. Correlation coefficients (Pearson/Spearman) were used to assess the correlation between all different parameters. A linear regression was performed to quantify the strength of the correlation $\left(R^{2}\right)$ for both groups studied. A receiver operating characteristic (ROC) curve analysis was performed in order to obtain the accuracy of the four measurements. A ROC curve is a graphical plot that reveals the effectiveness of a binary classifier system as its differentiation threshold changes. The curve is created by charting the true positive rate against the false positive rate at various threshold settings. The accuracy of the analysis comes defined according to the level of classification of the tested group between two groups (with or without keratoconus). The area under the ROC curve measures the accuracy: 1 represents a perfect test, and 0.5 indicates a worthless test. A rough guide for classifying the accuracy of a diagnostic test is the traditional academic point system [34]: excellent if $0.90-1$; good if $0.80-0.90$, fair if $0.70-0.80$, and poor if $0.60-0.70$. Statistical analyses were performed using Prism (GraphPad, v6, La Jolla, CA, USA) and SPSS (SPSS Inc., v17.0, Chicago, IL, USA) software. 


\section{Results}

This study included 55 healthy eyes that did not present any ocular pathology [2], constituting 30 females $(54.5 \%)$ and 25 males $(45.5 \%)$, and 52 eyes diagnosed with keratoconus (KC) in grade I [35] formed by 27 females (51.9\%) and 25 males (48.1\%).

Table 1 shows the morphological, refractive, and visual outcomes in the two groups of eyes analysed during the study. No statistically significant differences between groups were found in anterior chamber depth $(p=0.28$, Mann-Whitney test), axial length $(p=0.29$, Student's $t$-test), white-to-white corneal diameter ( $p=0.69$, Mann-Whitney test), and age ( $p=0.08$, Mann-Whitney test). Significant differences between groups were found in the remaining visual, refractive, and anatomical parameters evaluated ( $p<0.01$ Student's $t$ and Mann-Whitney tests). Additionally, note that the calculated effect sizes for each group allows quantifying the degree of change.

Table 1. Main clinical features of the healthy and keratoconus (stage I-AK) groups analysed in the study.

\begin{tabular}{|c|c|c|c|}
\hline \multirow{2}{*}{ Clinical Feature } & Healthy Group $(N=55)$ & Keratoconus Group $(N=52)$ & \multirow{2}{*}{$p$ Value } \\
\hline & $(\mathrm{M} \pm \mathrm{SD})$ & $(\mathrm{M} \pm \mathrm{SD})$ & \\
\hline Age (years) & $37.01 \pm 15.11$ & $39.11 \pm 16.7$ & 0.08 \\
\hline (ES) & - & 1.29 & - \\
\hline Axial Length (mm) & $24.01 \pm 1.61$ & $24.07 \pm 1.39$ & 0.29 \\
\hline (ES) & - & 1.19 & - \\
\hline Corrected Distance Visual Acuity & $0.98 \pm 0.09$ & $0.04 \pm 0.61$ & 0.0001 \\
\hline$(\mathrm{ES})$ & - & -0.11 & - \\
\hline Sphere (D) & $-0.49 \pm 3.31$ & $-0.91 \pm 1.36$ & 0.0001 \\
\hline (ES) & - & -0.31 & - \\
\hline Cylinder (D) & $-0.79 \pm 1.07$ & $-2.01 \pm 1.29$ & 0.0001 \\
\hline (ES) & - & -1.10 & - \\
\hline Spherical equivalent (D) & $-0.96 \pm 3.31$ & $-1.91 \pm 1.47$ & 0.0001 \\
\hline$(\mathrm{ES})$ & - & -0.90 & - \\
\hline $\begin{array}{l}\text { Minimal corneal thickness }(\mu \mathrm{m}) \\
(\mathrm{ES})\end{array}$ & $\begin{array}{c}540.07 \pm 31.98 \\
-\end{array}$ & $\begin{array}{l}469.68 \pm 34.01 \\
-0.12\end{array}$ & $\begin{array}{c}0.0001 \\
-\end{array}$ \\
\hline Central corneal thickness $(\mu \mathrm{m})$ & $542.98 \pm 32.99$ & $472.89 \pm 41.10$ & 0.0001 \\
\hline$(\mathrm{ES})$ & - & -0.20 & - \\
\hline Anterior chamber depth (mm) & $2.77 \pm 0.47$ & $3.41 \pm 0.4$ & 0.28 \\
\hline$(\mathrm{ES})$ & - & 0.10 & - \\
\hline White to white corneal diameter ( $\mathrm{mm}$ ) & $11.97 \pm 0.44$ & $12.02 \pm 0.50$ & 0.69 \\
\hline (ES) & - & 0.07 & - \\
\hline
\end{tabular}

$\mathrm{M}=$ mean, $\mathrm{SD}=$ standard deviation, $\mathrm{ES}=$ Effect size.

On the other hand, all of the morphogeometric variables showed differences between normal and keratoconic eyes, as seen in Table 2. Regarding area parameters, both sagittal plane areas (by passing through the posterior apex and the posterior minimum thickness point, respectively) were statistically higher in the subjects with healthy corneas. As expected, major deviations for the posterior apex and for the posterior minimum thickness point were also observed in the group of keratoconic corneas (see Table 2).

Statistically significant correlations between all the modelled morphogeometric variables for the normal group are summarized in Table 3, while Table 4 shows the significant correlations for the grade I-KC.

In the healthy eyes group, one strong correlation (above 0.9) was achieved between the sagittal plane apex area and the sagittal plane area at the minimum thickness point $(r=0.992 ; p<0.000)$. This correlation is also verified in the grade I-KC group $(r=0.998 ; p<0.000)$. 
Table 2. Morphogeometric variables measured in healthy and keratoconic (stage I-AK) corneas.

\begin{tabular}{|c|c|c|c|}
\hline \multirow[t]{2}{*}{ Parameter } & $\begin{array}{l}\text { Healthy Group } \\
\quad(N=55)\end{array}$ & $\begin{array}{l}\text { Keratoconus Group } \\
\qquad(N=52)\end{array}$ & \multirow[t]{2}{*}{$p$ Value } \\
\hline & $(\mathrm{M} \pm \mathrm{SD})$ & $(\mathrm{M} \pm \mathrm{SD})$ & \\
\hline Sagittal plane apex area $\left(\mathrm{mm}^{2}\right)$ & $4.35 \pm 0.31$ & $3.92 \pm 0.31$ & 0.0001 \\
\hline (ES) & - & 1.21 & - \\
\hline Sagittal plane area at minimum thickness point $\left(\mathrm{mm}^{2}\right)$ & $4.34 \pm 0.30$ & $3.90 \pm 0.29$ & 0.0001 \\
\hline$(\mathrm{ES})$ & - & 1.18 & - \\
\hline Posterior apex deviation (mm) & $0.08 \pm 0.03$ & $0.19 \pm 0.11$ & 0.0001 \\
\hline (ES) & - & -1.19 & - \\
\hline Posterior minimum thickness point deviation (mm) & $0.80 \pm 0.26$ & $1.02 \pm 0.35$ & 0.0001 \\
\hline (ES) & - & -0.88 & - \\
\hline
\end{tabular}

Table 3. Correlations between parameters for the normal group.

\begin{tabular}{ccccc}
\hline Parameter & $\begin{array}{c}\text { Sagittal Plane } \\
\text { Apex Area }\end{array}$ & $\begin{array}{c}\text { Sagittal Plane Area at } \\
\text { Minimum Thickness Point }\end{array}$ & $\begin{array}{c}\text { Posterior Apex } \\
\text { Deviation }\end{array}$ & $\begin{array}{c}\text { Posterior Minimum } \\
\text { Thickness Point Deviation }\end{array}$ \\
\hline Sagittal plane apex area & $r=1$ & $\begin{array}{c}r=\mathbf{0 . 9 9 2} \\
p<0.000\end{array}$ & $\begin{array}{c}r=0.16 \\
p<0.001\end{array}$ & $\begin{array}{c}r=0.04 \\
p<0.001\end{array}$ \\
\hline $\begin{array}{c}\text { Sagittal plane area at } \\
\text { minimum thickness point }\end{array}$ & $r=\mathbf{0 . 9 9 2}$ & $r=1$ & $r=0.124$ & $r=-0.18$ \\
\hline Posterior apex deviation & $r=0.000$ & $r=0.124$ & $r=1$ & $r=0.711$ \\
Posterior minimum & $p<0.001$ & $p<0.000$ & $r=0.711$ & $p<0.000$ \\
thickness point deviation & $p=0.04$ & $r=-0.18$ & $p<0.000$ & $r=1$ \\
\hline
\end{tabular}

Table 4. Correlations between parameters for the grade I-KC group.

\begin{tabular}{ccccc}
\hline Parameter & $\begin{array}{c}\text { Sagittal Plane } \\
\text { Apex Area }\end{array}$ & $\begin{array}{c}\text { Sagittal Plane Area at } \\
\text { Minimum Thickness Point }\end{array}$ & $\begin{array}{c}\text { Posterior Apex } \\
\text { Deviation }\end{array}$ & $\begin{array}{c}\text { Posterior Minimum } \\
\text { Thickness Point Deviation }\end{array}$ \\
\hline Sagittal plane apex area & $r=1$ & $\begin{array}{c}r=0.998 \\
p<0.000\end{array}$ & $\begin{array}{c}r=-0.376 \\
p<0.000\end{array}$ & $\begin{array}{c}r=-0.280 \\
p<0.002\end{array}$ \\
\hline $\begin{array}{c}\text { Sagittal plane area at } \\
\text { minimum thickness point }\end{array}$ & $\begin{array}{c}r=\mathbf{0 . 9 9 8} \\
p<0.000\end{array}$ & $r=1$ & $r=-0.379$ & $p<0.000$ \\
\hline Posterior apex deviation & $r=-0.376$ & $r=-0.379$ & $r=1$ & $r=0.481$ \\
Posterior minimum & $p<0.000$ & $p<0.000$ & $r=0.481$ & $p<0.000$ \\
thickness point deviation & $p=-0.280$ & $r=-0.294$ & $p<0.000$ & $r=1$ \\
\hline
\end{tabular}

The predictive value of the modelled variables was established by a ROC analysis. The geometric variables analysed during the study present the following results in the diagnosis of the disease (Figure 5): Sagittal plane apex area (area under the ROC curve: $0.858, p<0.0001$, std. error: 0.039, 95\% confidence interval: $0.772-0.917$ ), with a cut-off value of $4.274 \mathrm{~mm}^{2}$, and an associated sensitivity and specificity of $57.04 \%$ and $92.01 \%$, respectively; posterior apex deviation (area under the ROC curve: 0.918, $p<0.0001$, std. error: $0.038,95 \%$ confidence interval: $0.849-0.988$ ), with a cut-off value of $0.099 \mathrm{~mm}$, and an associated sensitivity and specificity of $90.01 \%$ and $88.41 \%$, respectively; sagittal plane area at minimum thickness point (area under the ROC curve: 0.853, $p<0.0001$, std. error: 0.039, 95\% confidence interval: 0.780-0.932), with a cut-off value of $4.266 \mathrm{~mm}^{2}$, and an associated sensitivity and specificity of $62.02 \%$ and $91.07 \%$, respectively; posterior minimum thickness point deviation (area under the ROC curve: 0.694, $p<0.0001$, std. error: $0.059,95 \%$ confidence interval: $0.577-0.812$ ), with a cut-off value of $0.0855 \mathrm{~mm}$, and an associated sensitivity and specificity of $63.1 \%$ and $91.11 \%$, respectively. 


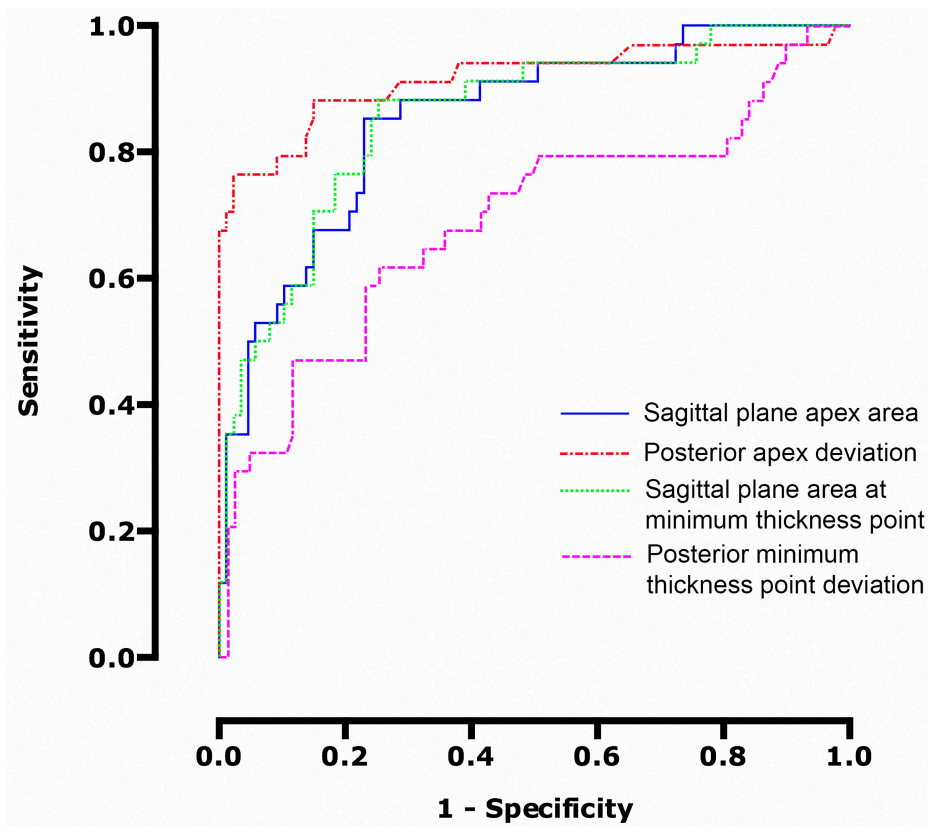

Figure 5. ROC curve modelling sensitivity versus 1-specificity for the variables analysed.

Thus, according to the area under the curve variable calculated for the analysed parameters, it was concluded that posterior apex deviation is the parameter that provides a higher rate of discrimination between normal and keratoconus grade I corneas. Nonetheless, other relevant statistical differences between healthy and diseased eyes are present, and most of the variables studied differ between groups. All these results make it possible to differentiate, with high sensitivity and specificity, healthy corneas from those patients diagnosed with keratoconus grade I.

\section{Discussion}

In ophthalmology, recognition and analysis of geometric patterns have become important tools in identifying diseases, for instance, for keratoconus diagnosis. The advantage of morphogeometric pattern recognition identification is that it reduces the variability created by less relevant variables, making the process depend only of those variables that are strong enough to support the model. Thus, this type of approach may be beneficial for a corneal pathology, like keratoconus, which is considered to be a rare disease [4].

However, while still being considered a rare disease, its diagnosis is made more frequently today than before due to the progress of corneal diagnostic imaging technology (such as Scheimpflug tomography) and, especially, its routine application to patients that are candidates for refractive surgery with no visual symptoms and normal vision harbouring initial stages of this disease. Such initial cases are considered as major contraindications for corneal refractive surgery. The differentiation between normal and abnormal patterns of corneal topography is, today, well sustained by the definition of different corneal topography and corneal aberrometry indices and corneal volumetric and densitometry data, among others [36]. However, the geometrical characterization indices proposed by these devices are not easily compatible between different tomographers, generating confusion in the ophthalmic community [8].

In the literature, some works that use CAD models integrate the patient-specific model concept, which provides quantitative results applicable to a specific patient [16-20]. This model is obtained from the so called "raw data" [21], which are generated by systems based on the projection of a slit light onto the cornea and on the principle of Scheimpflug photography [5].

Several works in the finite elements field use these raw data for several purposes: to predict the response to refractive surgeries [19] or the response to the intrastromal ring segment implantation 
in corneas with keratoconus [18], to analyse non-surgical corneal modifications, such as applanation tonometry for intraocular pressure measurement [20], or to analyse the behaviour of corneal tissue properties in different scenarios [16,17]. In all these cases, raw data were provided by the Pentacam (Oculus Optikgeräte GmbH, Wetzlar, Germany) [16,18], Sirius (CSO, Florence, Italy) [19,20], or Galilei (Ziemer Ophthalmic Systems AG, Port, Switzerland) [17] devices. However, due to extrinsic errors [5] occurring during the measurement process, the generated raw data were incomplete; thus, the authors of the mentioned works were forced to interpolate with the aim of obtaining a complete representation of the corneal surfaces and, thus, generate a 3D model of the cornea for its posterior use in the finite elements method.

In the present study, raw data obtained from the Sirius (CSO, Florence, Italy) device was also used. These data also presented the previously-mentioned extrinsic errors. However, the authors adopted a design protocol based on geometrical and clinical principles in which no interpolation was performed, using only real data [33]. Thus, the geometric model generated was authentic and completely personalized of the morphological biometry of the cornea, with a high sensitivity for the diagnosis and posterior analysis of the progression of the disease.

In the case of keratoconus, the deterioration process of the corneal structure is characterised by a significant reduction of the corneal thickness in comparison to healthy eyes. This is triggered by an alteration in corneal collagen fibres causing stromal thinning and breaks in the Bowman's membrane during the different stages of the disease [4,37]. Furthermore, the presence of corneal irregularities and the influence of the intraocular pressure on these weakened structures will create local steepening and an increased radius of curvature, which will lead to an increased posterior corneal surface area $[3,4,37,38]$. In the keratoconus group, the sagittal plane areas were smaller because of their local structural weakening due to fewer collagen fibres in each lamella and the influence of the intraocular pressure [3]. These values are consistent with those published by different studies $[38,39]$ which have reported that, in irregular corneal areas, the geometry of posterior corneal surfaces were affected due to the lower number of stromal lamellae and the smaller lamellar interconnection. In other studies, where a similar characterisation of the corneas has been described for the differentiation of pathologic eyes, the variables related to corneal thickness and volume, among others, are directly given by the software of the topographers $[40,41]$. However, in the present study, the morphogeometric variables are calculated from a 3D model generated using only real and non-interpolated raw point cloud data provided by the tomographer.

The average distance from the apex of the posterior corneal surface to the Z-axis (posterior apex deviation) differed between groups, with the largest deviations found in the group of eyes with keratoconus. The deviation of the apex on the posterior surface of the cornea was larger in the eyes with keratoconus $(0.19 \pm 0.11 \mathrm{~mm})$, and there was also a slight deviation in healthy corneas $(0.08 \pm 0.03 \mathrm{~mm}$, effect size: -1.19$)$ according to the toricity manifested in the subjective refraction [35]. The aforementioned presence of an irregular corneal surface, which created a protrusion in the keratoconic eye, also led to an increased corneal curvature [42] and, therefore, to an increase in the deviation of the point of minimum thickness (maximum curvature) of the posterior corneal surface (average distance from the minimum thickness point of the posterior surface to the Z-axis). These deviations were greater in the eyes with keratoconus $(1.02 \pm 0.35 \mathrm{~mm})$ compared with healthy eyes $(0.80 \pm 0.26 \mathrm{~mm}$, effect size: -0.88$)$. This fact reveals that, even though the curvature radii are greater on posterior surfaces in the $\mathrm{KC}$ group, the morphogeometric variables register the tendency of the cornea to develop and to maintain its structure in the form of a meniscus in the early grades of the disease.

This trend is in line with the tendency reported by other studies [43], where the posterior radius of curvature is analysed for groups with healthy eyes and keratoconic eyes according to the Amsler-Krumeich classification. Specifically for keratoconus, in this study a significant increase of the curvature of the posterior corneal surface was observed with respect to the anterior surface $(p<0.01)$ 
in the early grades of the disease. Some researchers have evaluated certain corneal irregularity ratios and concluded that they were higher for keratoconic corneas [44,45].

The analysis of these variables concluded that the parameter that provides a higher discrimination rate between normal corneas and corneas with keratoconus was the posterior apex deviation (ROC area: 0.918, $p<0.0001$, std. error: $0.038,95 \%$ CI: $0.849-0.988$ ), with a cut-off value of 0.099 $\mathrm{mm}$, and an associated sensitivity and specificity of $90.01 \%$ and $88.41 \%$, respectively. This is justified due to the structural instability that keratoconic corneas present in its architecture, the posterior surface being the most susceptible to variations given the forces exerted on the tissue, so the posterior curvature might influence the visual function. Accordingly, we hypothesize that the magnitude of the posterior apex deviation represents the best performance to recognize the geometric profile of the KC stage where the visual acuity starts to impair. Several studies have concluded the importance of, and interest in, the posterior corneal surface [45]. However, to the authors' knowledge, this is the first study to describe this phenomenon in the disease stage when the degree of corneal protrusion is apparent in the posterior corneal surface, which supports the diagnosis accuracy of the proposed method. Furthermore, a satisfactory level of discriminative ability for the sagittal plane apex area (ROC area: 0.858), sagittal plane area at the minimum thickness point (ROC area: 0.853), and the posterior minimum thickness point deviation (ROC area: 0.694) parameters were found.

A comparison of the screening of keratoconus [46] provided by the Sirius topographer and the detection of corneal ectasia using the posterior apex deviation was performed. If we consider the two possibilities of the screening of keratoconus from the Sirius topographer, two groups are differentiated: normal eyes and keratoconus-compatible (keratoconus and borderline). From these sample data of keratoconus the Sirius topographer considered $19.4 \%$ normal eyes, and $80.6 \%$ keratoconus-compatible. If we consider the posterior apex deviation as the best parameter for screening keratoconus in the morphogeometric model due to the best ROC analysis, two groups are differentiated based in the percentiles obtained: normal eyes were considered as the mean value of posterior apex deviation less than 0.1 (the 75th percentile of the normal sample), and keratoconus, when the mean value of posterior apex deviation was higher than 0.11 (the 75th percentile of keratoconus-compatible eyes). Regarding this classification based on the posterior apex deviation, the following results were obtained: $16.1 \%$ normal eyes, $83.9 \%$ keratoconus-compatible. These outcomes indicate that the morphogeometric model and the Sirius screening are comparable. Furthermore, the morphogeometric model is able to discriminate between keratoconus-compatible and normal eyes approximately $3 \%$ more than the Sirius topographer. When the sensitivity and specificity of the keratoconus screening of the Sirius topographer were calculated from our sample, a sensitivity of $80.6 \%$ and a specificity of $97.7 \%$ were obtained, in comparison with a sensitivity and specificity of $90.01 \%$ and $88.41 \%$, respectively, for the posterior apex deviation. This fact indicates that the morphogeometric model is able to identify keratoconus with success in comparison with the Sirius topographer. However, this model is less predictable to identify eyes without keratoconus than the keratoconus screening from the Sirius device. In order to improve the specificity outcomes, future studies combining these morphogeometric parameters with optic variables are required.

\section{Conclusions}

In summary, this new concept is based on the recognition (at the local level) of determined geometrical patterns defined on the posterior corneal surface and, more specifically, on the region where the first structural anomalies are developed because of the manifestation of the disease. These deformations lead to a deterioration of the visual quality of the patients during the disease progression, in agreement with the main conclusions drawn by the group of world experts in keratoconus. For this purpose, the method proposed in this work analyses certain parametric morphogeometric operators extracted from a custom patient-specific 3D model, providing a feasible and new diagnostic approach of the keratoconus. Furthermore, and under the concept of interoperability [47], this non-invasive clinical diagnosis method could be used by current corneal 
topographers that are based on the projection of a slit of light onto the cornea and on the principle of Scheimpflug photography. This could lead to a better understanding of the ethology and prognosis of this eye disease.

Acknowledgments: This work was supported by the Thematic Network for Co-Operative Research in Health (RETICS-RD16/0008/0012), financed by the Carlos III Health Institute and the European Regional Development Fund (FEDER).

Author Contributions: Laurent Bataille, Francisco Cavas-Martínez and Daniel G. Fernández-Pacheco designed the experiments, which were later executed by Laurent Bataille and Jorge L. Alio. The analysis of the obtained data was performed by Francisco Cavas-Martínez, Daniel G. Fernández-Pacheco, Francisco J. F. Cañavate and Laurent Bataille. Finally, all authors contributed to the writing of the manuscript.

Conflicts of Interest: The authors declare no conflict of interest.

\section{References}

1. Eghrari, A.O.; Riazuddin, S.A.; Gottsch, J.D. Overview of the cornea: Structure, function, and development. Prog. Mol. Biol. Transl. Sci. 2015, 134, 7-23. [PubMed]

2. Montalban, R.; Alio, J.L.; Javaloy, J.; Pinero, D.P. Correlation of anterior and posterior corneal shape in keratoconus. Cornea 2013, 32, 916-921. [CrossRef] [PubMed]

3. Piñero, D.P.; Alio, J.L.; Barraquer, R.I.; Michael, R.; Jimenez, R. Corneal biomechanics, refraction, and corneal aberrometry in keratoconus: An integrated study. Investig. Ophthalmol. Vis. Sci. 2010, 51, 1948-1955. [CrossRef] [PubMed]

4. Rabinowitz, Y.S. Keratoconus. Surv. Ophthalmol. 1998, 42, 297-319. [CrossRef]

5. Cavas-Martinez, F.; De la Cruz Sanchez, E.; Nieto Martinez, J.; Fernandez Canavate, F.J; Fernandez-Pacheco, D.G. Corneal topography in keratoconus: State of the art. Eye Vis. 2016, 3, 5. [CrossRef] [PubMed]

6. Randleman, J.B.; Russell, B.; Ward, M.A.; Thompson, K.P.; Stulting, R.D. Risk factors and prognosis for corneal ectasia after lasik. Ophthalmology 2003, 110, 267-275. [CrossRef]

7. Dubbelman, M.; Sicam, V.A.; Van der Heijde, G.L. The shape of the anterior and posterior surface of the aging human cornea. Vis. Res. 2006, 46, 993-1001. [CrossRef] [PubMed]

8. Hernandez-Camarena, J.C.; Chirinos-Saldana, P.; Navas, A.; Ramirez-Miranda, A.; de la Mota, A.; Jimenez-Corona, A.; Graue-Hernindez, E.O. Repeatability, reproducibility, and agreement between three different scheimpflug systems in measuring corneal and anterior segment biometry. J. Refract. Surg. 2014, 30, 616-621. [CrossRef] [PubMed]

9. Gomes, J.A.; Tan, D.; Rapuano, C.J.; Belin, M.W.; Ambrosio, R., Jr.; Guell, J.L.; Malecaze, F.; Nishida, K.; Sangwan, V.S. Global consensus on keratoconus and ectatic diseases. Cornea 2015, 34, 359-369. [CrossRef] [PubMed]

10. Auer, M.; Stollberger, R.; Regitnig, P.; Ebner, F.; Holzapfel, G.A. In vitro angioplasty of atherosclerotic human femoral arteries: Analysis of the geometrical changes in the individual tissues using mri and image processing. Ann. Biomed. Eng. 2010, 38, 1276-1287. [CrossRef] [PubMed]

11. Gayzik, F.S.; Moreno, D.P.; Danelson, K.A.; McNally, C.; Klinich, K.D.; Stitzel, J.D. External landmark, body surface, and volume data of a mid-sized male in seated and standing postures. Ann. Biomed. Eng. 2012, 40, 2019-2032. [CrossRef] [PubMed]

12. Arokiaraj, M.C.; De Santis, G.; De Beule, M.; Palacios, I.F. A novel tram stent method in the treatment of coronary bifurcation lesions-Finite element study. PLoS ONE 2016, 11, e0149838. [CrossRef] [PubMed]

13. Zhao, S.; Gu, L.; Froemming, S.R. On the importance of modeling stent procedure for predicting arterial mechanics. J. Biomech. Eng. 2012, 134, 121005. [CrossRef] [PubMed]

14. Lohfeld, S.; Barron, V.; McHugh, P.E. Biomodels of bone: A review. Ann. Biomed. Eng. 2005, 33, 1295-1311. [CrossRef] [PubMed]

15. Wang, Y.T.; Yang, X.J.; Yan, B.; Zeng, T.H.; Qiu, Y.Y.; Chen, S.J. Clinical application of three-dimensional printing in the personalized treatment of complex spinal disorders. Chin. J. Traumatol. 2016, 19, 31-34. [CrossRef] [PubMed]

16. Ariza-Gracia, M.Á.; Redondo, S.; Piñero Llorens, D.; Calvo, B.; Rodriguez Matas, J.F. A predictive tool for determining patient-specific mechanical properties of human corneal tissue. Comput. Methods Appl. Mech. Eng. 2017, 317, 226-247. [CrossRef] 
17. Asher, R.; Gefen, A.; Moisseiev, E.; Varssano, D. An analytical approach to corneal mechanics for determining practical, clinically-meaningful patient-specific tissue mechanical properties in the rehabilitation of vision. Ann. Biomed. Eng. 2015, 43, 274-286. [CrossRef] [PubMed]

18. Lago, M.A.; Ruperez, M.J.; Monserrat, C.; Martinez-Martinez, F.; Martinez-Sanchis, S.; Larra, E.; Diez-Ajenjo, M.A.; Peris-Martinez, C. Patient-specific simulation of the intrastromal ring segment implantation in corneas with keratoconus. J. Mech. Behav. Biomed. Mater. 2015, 51, 260-268. [CrossRef] [PubMed]

19. Simonini, I.; Pandolfi, A. Customized finite element modelling of the human cornea. PLoS ONE 2015, 10, e0130426. [CrossRef] [PubMed]

20. Simonini, I.; Pandolfi, A. The influence of intraocular pressure and air jet pressure on corneal contactless tonometry tests. J. Mech. Behav. Biomed. Mater. 2016, 58, 75-89. [CrossRef] [PubMed]

21. Ramos-Lopez, D.; Martinez-Finkelshtein, A.; Castro-Luna, G.M.; Pinero, D.; Alio, J.L. Placido-based indices of corneal irregularity. Optom. Vis. Sci. Off. Publ. Am. Acad. Optom. 2011, 88, 1220-1231. [CrossRef] [PubMed]

22. Klyce, S.D.; Karon, M.D.; Smolek, M.K. Advantages and disadvantages of the zernike expansion for representing wave aberration of the normal and aberrated eye. J. Refract. Surg. 2004, 20, S537-S541. [PubMed]

23. Ares, M.; Royo, S. Comparison of cubic B-spline and zernike-fitting techniques in complex wavefront reconstruction. Appl. Opt. 2006, 45, 6954-6964. [CrossRef] [PubMed]

24. Piñero, D.P.; Nieto, J.C.; Lopez-Miguel, A. Characterization of corneal structure in keratoconus. J. Cataract Refract. Surg. 2012, 38, 2167-2183. [CrossRef] [PubMed]

25. Browning, J.E.; McMann, A.K. Computational Engineering: Design, Development and Applications; Nova Science Publishers: Hauppauge, NY, USA, 2012.

26. Espinosa, J.; Mas, D.; Perez, J.; Illueca, C. Optical surface reconstruction technique through combination of zonal and modal fitting. J. Biomed. Opt. 2010, 15, 026022. [CrossRef] [PubMed]

27. Piegl, L.A.; Tiller, W. Approximating surfaces of revolution by nonrational B-splines. IEEE Comput. Graph. Appl. 2003, 23, 46-52. [CrossRef]

28. Malve, M.; Gharib, A.M.; Yazdani, S.K.; Finet, G.; Martinez, M.A.; Pettigrew, R.; Ohayon, J. Tortuosity of coronary bifurcation as a potential local risk factor for atherosclerosis: CFD steady state study based on in vivo dynamic ct measurements. Ann. Biomed. Eng. 2015, 43, 82-93. [CrossRef] [PubMed]

29. Fisk, J.P.; Wayne, J.S. Development and validation of a computational musculoskeletal model of the elbow and forearm. Ann. Biomed. Eng. 2009, 37, 803-812. [CrossRef] [PubMed]

30. Benítez, H.A.; Püschel, T.A. Modelling shape variance: Geometric morphometric applications in evolutionary biology. Int. J. Morphol. 2014, 32, 998-1008. [CrossRef]

31. Klingenberg, C. Analyzing fluctuating asymmetry with geometric morphometrics: Concepts, methods, and applications. Symmetry 2015, 7, 843-934. [CrossRef]

32. Cavas-Martínez, F.; Fernández-Pacheco, D.G.; De La Cruz-Sánchez, E.; Martínez, J.N.; Cañavate, F.J.F.; Alio, J.L. Virtual biomodelling of a biological structure: The human cornea. Dyna 2015, 90, 647-651.

33. Cavas-Martinez, F.; Fernandez-Pacheco, D.G.; De la Cruz-Sanchez, E.; Nieto Martinez, J.; Fernandez Canavate, F.j.; Vega-Estrada, A.; Plaza-Puche, A.B.; Alio, J.L. Geometrical custom modeling of human cornea in vivo and its use for the diagnosis of corneal ectasia. PLoS ONE 2014, 9, e110249. [CrossRef] [PubMed]

34. Bozikov, J.; Lijana, Z. Test Validity Measures and Receiver Operating Characteristic (Roc) Analysis. In Methods and Tools in Public Health: A Handbook for Teachers, Researchers and Health Professionals; Hans Jacobs Publishing Company: Lage, Germany, 2010; pp. 749-770.

35. Montalban, R.; Pinero, D.P.; Javaloy, J.; Alio, J.L. Correlation of the corneal toricity between anterior and posterior corneal surfaces in the normal human eye. Cornea 2013, 32, 791-798. [CrossRef] [PubMed]

36. Alió, J.; Vega-Estrada, A.; Sanz-Díez, P.; Peña-García, P.; Luisa Durán-García, M.; Maldonado, M. Keratoconus management guidelines. Int. J. Keratoconus Ect. Corneal Dis. 2015, 4, 1-39. [CrossRef]

37. Parker, J.S.; van Dijk, K.; Melles, G.R. Treatment options for advanced keratoconus: A review. Surv. Ophthalmol. 2015, 60, 459-480. [CrossRef] [PubMed]

38. Sherwin, T.; Brookes, N.H.; Loh, I.P.; Poole, C.A.; Clover, G.M. Cellular incursion into bowman's membrane in the peripheral cone of the keratoconic cornea. Exp. Eye Res. 2002, 74, 473-482. [CrossRef] [PubMed]

39. Ozgurhan, E.B.; Kara, N.; Yildirim, A.; Bozkurt, E.; Uslu, H.; Demirok, A. Evaluation of corneal microstructure in keratoconus: A confocal microscopy study. Am. J. Ophthalmol. 2013, 156, 885-893. [CrossRef] [PubMed] 
40. Cerviño, A.; Gonzalez-Meijome, J.M.; Ferrer-Blasco, T.; Garcia-Resua, C.; Montes-Mico, R.; Parafita, M. Determination of corneal volume from anterior topography and topographic pachymetry: Application to healthy and keratoconic eyes. Ophthalmic Physiol. Opt. 2009, 29, 652-660. [CrossRef] [PubMed]

41. Mannion, L.S.; Tromans, C.; O’Donnell, C. Reduction in corneal volume with severity of keratoconus. Curr. Eye Res. 2011, 36, 522-527. [CrossRef] [PubMed]

42. Ozcura, F.; Yildirim, N.; Tambova, E.; Sahin, A. Evaluation of goldmann applanation tonometry, rebound tonometry and dynamic contour tonometry in keratoconus. J. Optom. 2017, 10, 117-122. [CrossRef] [PubMed]

43. Montalban, R.; Alio, J.L.; Javaloy, J.; Pinero, D.P. Comparative analysis of the relationship between anterior and posterior corneal shape analyzed by scheimpflug photography in normal and keratoconus eyes. Graefe's Arch. Clin. Exp. 2013, 251, 1547-1555. [CrossRef] [PubMed]

44. Safarzadeh, M.; Nasiri, N. Anterior segment characteristics in normal and keratoconus eyes evaluated with a combined scheimpflug/placido corneal imaging device. J. Curr. Ophthalmol. 2016, 28, 106-111. [CrossRef] [PubMed]

45. Schlegel, Z.; Hoang-Xuan, T.; Gatinel, D. Comparison of and correlation between anterior and posterior corneal elevation maps in normal eyes and keratoconus-suspect eyes. J. Cataract Refract. Surg. 2008, 34, 789-795. [CrossRef] [PubMed]

46. Arbelaez, M.C.; Versaci, F.; Vestri, G.; Barboni, P.; Savini, G. Use of a support vector machine for keratoconus and subclinical keratoconus detection by topographic and tomographic data. Ophthalmology 2012, 119, 2231-2238. [CrossRef] [PubMed]

47. ISO/IEC/IEEE International Standard. Systems and Software Engineering —Life Cycle Processes—Requirements Engineering; ISO/IEC/IEEE: New York, NY, USA, 2011; pp. 1-94.

(C) 2017 by the authors. Licensee MDPI, Basel, Switzerland. This article is an open access article distributed under the terms and conditions of the Creative Commons Attribution (CC BY) license (http:/ / creativecommons.org/licenses/by/4.0/). 